IL21-expressing T follicular (Tfh) and peripheral helper (Tph) cells measured by FC. These generated T cells were also cultured in the presence of memory $B$ cells. After 5 days of co-culture, plasmablast generation and Ig levels were assessed by FC and ELISA, respectively. Inhibition of OX40-OX40L interaction in vitro was achieved using ISB 830, a novel anti-OX40 mAb currently used in clinical trials.

Results: Among the co-stimulatory molecules tested, percentages of $\mathrm{OX}_{40 \mathrm{~L}^{+} \text {neu- }}$ trophils in SLE $(n=54)$ were increased compared to HD $(n=25)($ mean + SD: HD = $1,34 \% \pm 1.62$ vs SLE $=4,53 \% \pm 8.1 ; p=0.29$ ). OX40L expression positively correlated with SLE disease activity score (SLEDAI) $(p=0,04 ; r=0,31)$ and with anti-DNA antibodies $(p=0,04, r=0,33)$. Of note, the percentage of $\mathrm{OX}_{40 \mathrm{~L}^{+} \text {neutrophils was }}$ higher in anti-sm-RNP ${ }^{+}$patients $(n=16$, mean $=9 \% \pm 9.8)$, compared to anti-smRNP' patients $(n=27$, mean $=1,4 \% \pm 2.5 ; p=0,02)$. The percentage of $\mathrm{OX}_{40 \mathrm{~L}^{+}}$ neutrophils was higher in patients with class III or IV lupus nephritis, and inflammatory infiltrate within the kidney biopsy disclosed $\mathrm{OX}_{40 \mathrm{~L}^{+}}$neutrophils, in close contact with T cells. Neutrophils from HD express OX40L with TLR8 agonist, or IFNa priming followed by TLR7 agonist. When memory CD4 T cells were cultured in the presence of TLR8-stimulated neutrophils, the proportion of IL21-expressing Tfh $\left(\mathrm{CXCR} 5^{+} \mathrm{PD} 1^{+}\right)$and Tph $\left(\mathrm{CXCR} 5^{-} \mathrm{PD} 1^{\mathrm{hi}}\right)$ were increased, compared to culture with unstimulated neutrophils. This process was dependent on OX40-OX40L interactions, since in vitro treatment with the anti-OX40 blocking antibody ISB 830, inhibited the differentiation of memory T cells into Tfh and Tph. Both generated Tfh and Tph were able to promote the differentiation of memory B cells into lg-secreting plasmablasts.

Conclusion: Our results disclose an unprecedented phenomenon where cross-talk between TLR7/8-activated neutrophils and CD4 lymphocytes operates through OX40L-OX40 costimulation, and neutrophils promote the differentiation of pro-inflammatory Tfh and Tph, as well as IL21 production. Therefore, OX40L/OX40 should be considered as a potentially therapeutic axis in SLE patients.

References:

[1] Jacquemin et al. Immunity 2015 ;

[2] Jacquemin et al. JCI Insight 2018

Disclosure of Interests: Angela Pappalardo Grant/research support from: Ichnos Sciences, Elodie Wojciechowski: None declared, Itsaso Odriozola: None declared, Isabelle Douchet: None declared, Nathalie Merillon: None declared, Andrea Boizard-Moracchini: None declared, Pierre Duffau: None declared, Estibaliz Lazaro: None declared, Marie-Agnes Doucey Employee of: Ichnos Sciences, Lamine Mbow Employee of: Ichnos Sciences, Christophe Richez Consultant of: Abbvie, Amgen, Mylan, Pfizer, Sandoz and UCB., Patrick Blanco Grant/research support from: Ichnos Sciences

DOI: 10.1136/annrheumdis-2020-eular.2739

\section{THU0036 \\ FIRST-IN-HUMAN TRIAL OF BCMA-CD19 COMPOUND CAR IN THE TREATMENT OF AUTOANTIBODY MEDIATED DISORDERS}

F. Liu ${ }^{1}$, H. Zhang ${ }^{2}$, X. Wang ${ }^{1}$, J. Feng ${ }^{2}$, Y. $\mathrm{Cao}^{3}$, Y. Su${ }^{1}$, M. Wada ${ }^{3}$, Y. Ma ${ }^{4}$, Y. Ma ${ }^{3} .{ }^{1}$ Chengdu Military General Hospital, Hematology, Chengdu, China; ${ }^{2}$ Peking University Shenzhen Hospital, Shenzhen, China; ${ }^{3}$ iCell Gene Therapeutics LLC, Stony Brook, NY, United States of America; ${ }^{4}$ iCAR Bio Therapeutics Ltd, Zhongshan, China

Background: Donor-specific anti-HLA antibodies (DSAs) are antibodies in the recipient directed against donor class I/II HLA antigens. The existence of DSAs before allogenic hematopoietic stem cell transplantation (AHSCT) are known to cause primary graft failure. Currently there's no established method of DSA desensitization due to the long half-life of plasma cells.

Systemic lupus erythematosus (SLE) is a heterogeneous autoimmune disease involving in multiple organ systems mediated by numerous autoantibodies. Recent results have shown that depletion of B cells by CD19 CAR-T cells effectively reversed some manifestations in two SLE mouse models. However, plasma cells could be spared with single CD19 CAR-T cells, and peripheral circulating anti-DNA IgG and IgM autoantibodies remain elevated or increased in treated mice.

Objectives: We present the efficacy of BCMA-CD19 compound CAR (cCAR), which target on antibody- producing "root", both B cells and plasma cells in preclinical study and in our first-in-human phase 1 clinical trial.

Methods: We constructed a BCMA-CD19 cCAR composed of a complete BCMACAR fused to a complete CD19 CAR, separated by a self-cleaving P2A peptide. We assessed the functional activity of CCAR in co-culture assay with multiple cell lines. We also verified cCAR efficacy with two mouse models, injected with either BCMA-expressing MM.1S cells or CD19-expressing REH cells. In our phase 1 clinical trial, we enrolled patients with hematologic malignancies with antibody mediated disorders.

Results: BCMA-CD19 cCAR exhibited robust cytotoxic activity against the K562 cells engineered to express either CD19 or BCMA in co-culture assays, indicating the ability of each complete CAR domain to specifically lyse target cells. In mouse model study, cCAR-T cells were able to eliminate tumor cells in mice injected with MM.1S cells and REH cells, indicating that both BCMA and CD19 are specifically and equally lysing B cells and plasma cells in vivo, making BCMA-CD19 cCAR a candidate for clinical use.

In our first-in-human clinical trial, the first case is a 48 -year-old female patient having resistant B-ALL with high DSA titers. She exhibited complete remission of B-ALL at day 14 post-CAR T treatment. MFI of DSA dropped from 7800 to 1400 at 8 weeks post cCAR treatment, the reduction percentage was approximately $80 \%$ (Figure 1). The patient had no CRS, and no neurotoxicity was observed.

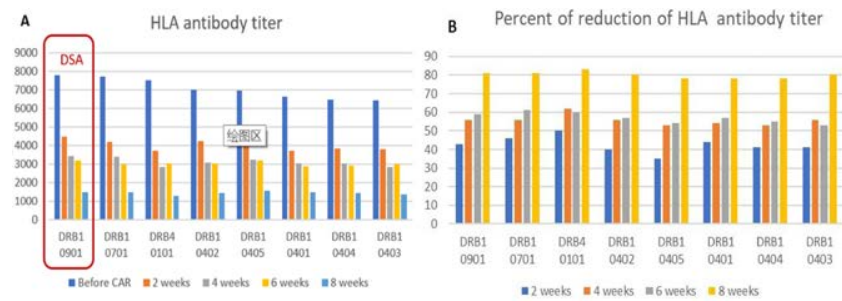

Figure 1. 1. A) MFI of DSA and other HLA antibodies before and at different time points after cCAR T infusion. B) the percent reduction post-transfusion of cCAR T cells at different time points.

The second case is a 41-year-old female patient having a refractory diffuse large $B$ cell lymphoma with bone marrow (BM) involvement. Furthermore, she has a 20 years of SLE, with manifestation of fever dependent of corticosteroids. On day 28 after cCAR treatment, PET/CT scan showed CR, and BM turned negative. In addition, she is independent of steroids, has no fever and other manifestations C3/C4 are within normal ranges, and all the ANA dropped significantly, especially the nuclear type ANA, which turned from $>1: 1000$ to be negative at day 64 . She had Grade 1 CRS but with no neurotoxicity observed. The absence of B cells and plasma cells persisted more than 5 months post CAR therapy.

Conclusion: Our first in human clinical trial on BCMA-CD19 cCAR demonstrated profound efficacy in reducing DSA levels in an AHSCT candidate and ANA titer in a SLE patient. There was strong clinical evidence of depletion of antibody-producing roots, B-cells and plasma cells in both patients. Our results further suggested that BCMA-CD19 CCAR has the potential to benefit patients receiving solid organ transplants or those with other antibody-mediated diseases.

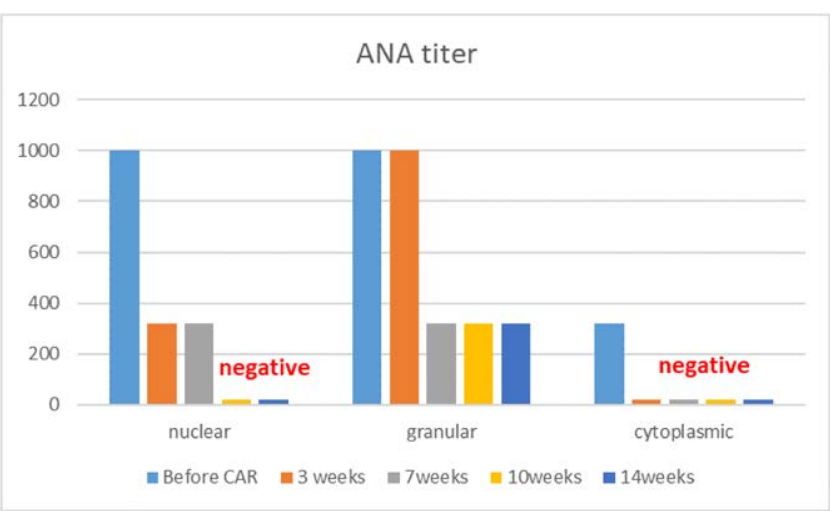

Figure 2. Reduction of different type of ANA titer at different time points.

Acknowledgments: patients and their families

Disclosure of Interests: Fang liu: None declared, Hongyu Zhang: None declared, Xiao Wang: None declared, Jia Feng: None declared, Yuanzhen cao Employee of: Employee of iCell Gene Therapeutics LLC, Yi Su: None declared, Masayuki Wada Employee of: employee of iCell Gene Therapeutics LLC, Yu Ma Employee of: employee of iCAR Bio Therapeutics Ltd, Yupo Ma Shareholder of: shareholder of iCell Gene Therapeutics LLC DOI: 10.1136/annrheumdis-2020-eular.4065

\begin{tabular}{|l|l}
\hline THU0037 & SURVIVIN INHIBITS TRANSCRIPTION OF PBX1 AND \\
& SUPPORTS THE EFFECTOR PHENOTYPE OF THE \\
MEMORY CD4 T CELLS IN RHEUMATOID ARTHRITIS
\end{tabular}

K. M. Andersson ${ }^{1}$, M. Erlandsson ${ }^{2}$, N. Oparina ${ }^{2}$, A. Damdimopoulos ${ }^{3}$, M. Jensen ${ }^{4}$, M. J. Garcia-Bonete ${ }^{4}$, G. Katona ${ }^{4}$, M. I. Bokarewa ${ }^{2} .{ }^{1}$ Göteborg university, Rheumatology and inflammation research, Göteborg, Sweden; ${ }^{2}$ the Sahlgrenska Academy at University of Gothenburg, Rheumatology and 